\title{
Mass spectrum analysis of membrane proteins reveals that CASK, CD36 and EPB42 are differentially expressed in pancreatic adenocarcinoma
}

\author{
MINGMING MENG ${ }^{1,2 *}$, SANHONG LIU ${ }^{3 *}$, CHEN WANG $^{4 *}$, XINJIN GU$^{5 *}$, \\ ENQIANG LINGHU ${ }^{1, * *}$ and XINYING XUE , *** $^{\text {,* }}$
}

\begin{abstract}
${ }^{1}$ Department of Gastroenterology and Hepatology, Chinese PLA General Hospital, Medical School of Chinese PLA, Beijing 100853; ${ }^{2}$ Department of Gastroenterology, Beijing Shijitan Hospital, Capital Medical University, Beijing 100038;

${ }^{3}$ Institute of Interdisciplinary Integrative Medicine Research, Shanghai University of Traditional Chinese Medicine,

Shanghai 201203; ${ }^{4}$ Shanghai Institute for Advanced Immunochemical Studies, ShanghaiTech University,

Shanghai 201210; ${ }^{5}$ Department of Hepatobiliary and Pancreatic Surgical Oncology,

Chinese PLA General Hospital, Beijing 100853; ${ }^{6}$ Department of Respiratory and Critical Care Medicine,

Beijing Shijitan Hospital, Capital Medical University, Beijing 100038, P.R. China
\end{abstract}

Received December 16, 2019; Accepted August 21, 2020

DOI: $10.3892 / \mathrm{ol} .2020 .12239$

\begin{abstract}
Pancreatic cancer is one of the most life-threatening malignancies worldwide. Despite advances in checkpoint immunotherapy for patients with cancer, the current immunotherapies have demonstrated limited benefits for the treatment of pancreatic cancer. Apart from the intricate microenvironments that restrict $\mathrm{T}$-cell function, membrane proteins other than programmed death-ligand 1 may also facilitate immune escape of tumor cells. The present study investigated the membrane proteins of seven paired pancreatic adenocarcinoma (PAAD) and adjacent normal tissues with mass spectrometry, and identified 10 up-and eight downregulated membrane proteins in PAAD. Together with the online database analysis, the results showed that the CASK protein was upregulated in PAAD samples and cell lines, and predicts poor outcomes in patients with PAAD. Furthermore, the results exhibited downregulated CD36 and EPB42 in PAAD
\end{abstract}

Correspondence to: Professor Enqiang Linghu, Department of Gastroenterology and Hepatology, Chinese PLA General Hospital, Medical School of Chinese PLA, 28 Fuxing Road, Beijing 100853, P.R. China

E-mail: linghuenqiang@vip.sina.com

Dr Xinying Xue, Department of Respiratory and Critical Care Medicine, Beijing Shijitan Hospital, Capital Medical University, 10 Tieyi St, Haidian, Beijing 100038, P.R. China

E-mail: xinyingxue2010@163.com

${ }^{*, \# C o n t r i b u t e d ~ e q u a l l y ~}$

Key words: mass spectrum, pancreatic cancer, membrane protein, CASK, CD36, EPB42 samples and cell lines, and higher levels of CD36. EPB42 was shown to predict improved survival outcomes in patients with PAAD. Overall, the results of the present study revealed PAAD-specific membrane proteins as potential diagnostic markers and drug-targets for the immunotherapy of pancreatic cancer.

\section{Introduction}

Checkpoint immunotherapy has emerged as a promising strategy for cancer treatments $(1,2)$ particularly for the treatment of melanoma (3) and lung cancer (4,5). Despite the attempts made to utilize checkpoint immunotherapy for pancreatic adenocarcinoma (PAAD) treatment, no clinical benefits have yet been observed $(6,7)$. It has been proposed that the specific immunosuppressive tumor microenvironment of pancreatic cancer is accountable for the limited clinical benefit of immunotherapy $(8,9)$. Immunosuppressive myeloid cells are one of the numerous barriers in immunotherapy for pancreatic cancer (10), as well as high stromal density (11). Hence, researchers established a combination of agents to facilitate checkpoint immunotherapy in pancreatic cancer. It has been demonstrated that the inhibition of interleukin 6 (IL-6) may enhance the efficacy of programmed death-ligand 1 (PD-L1) in pancreatic cancer (12). Dickkopf-3 neutralization also leads to an improvement in the immunotherapy effect in pancreatic cancer (13). However, there is the possibility that pancreatic cancer cells are able to utilize other cell surface markers in order to avoid checkpoint immunotherapy and thus facilitate disease progression.

The identification of cell surface markers for pancreatic cancer provides therapeutic and diagnostic strategies. Through the assistance of mass spectrometry, a cell surface proteoglycan, glypican-1, was found to be specifically enriched in 
pancreatic cancer cells and the associated exosomes (14), which provided a non-invasive diagnostic and screening tool to detect early stages of pancreatic cancer. Recently, it has been confirmed that plasminogen receptor S100A10 is enriched on the pancreatic cancer cell surface and contributes to cancer cell invasion (15). Consequently, it is important to characterize novel pancreatic cancer-specific cell surface markers that benefit immunotherapy by providing novel target sites and improve patient survival through facilitating curative surgical therapy via early diagnosis. To identify pancreatic cancer-specific cell surface markers, the present study utilized mass spectrometry and analyzed seven paired pancreatic adenocarcinoma tissues and adjacent tissues. The analysis led to the discovery of a novel panel of membrane proteins that predict the survival outcome of patients with pancreatic cancer, as well as potential diagnostic markers and therapeutic targets for pancreatic adenocarcinoma.

\section{Materials and methods}

PAAD samples. All patients involved in the present study provided written informed consent, and all procedures were approved by the Ethics Committee of Chinese PLA General Hospital (Beijing, China) in accordance with the Declaration of Helsinki. All PAAD and adjacent normal tissues $(\geq 10 \mathrm{~cm}$ from the tumor tissue) were obtained during surgeries between July and August 2017 at the Department of Hepatobiliary and Pancreatic Surgical Oncology. PAAD samples were confirmed by two blinded independent pathologists (with 10 years of experience with pathologic diagnosis of the pancreas) from the Department of Pathology at Chinese PLA General Hospital. The diagnostic criteria were based on clinical practice guidelines for pancreatic cancer issued by the National Comprehensive Cancer Network (16). PAAD and adjacent normal tissues were immediately dissected following surgery and directly frozen at $-80^{\circ} \mathrm{C}$. Patient information are listed in Table I.

Mass spectrometry (MS) analysis. PAAD and adjacent normal tissues were homogenized in a homogenizer (cat.no. JXSFTPRP-48; Shanghai Jingxin Medical Instruments Co., Ltd.) at $120 \mathrm{~Hz}$ for $2 \mathrm{~min}$, with 2, 2-mm steel beads in cell lysis buffer (50 mM Tris- $\mathrm{HCl}, \mathrm{pH} 7.5 ; 165 \mathrm{mM}$ sodium chloride; $10 \mathrm{mM}$ EDTA; $10 \mu \mathrm{g} / \mathrm{ml}$ aprotinin; $10 \mu \mathrm{g} / \mathrm{ml}$ leupeptin, and $1 \%$ NP-40). Homogenized samples were lyophilized overnight at $4^{\circ} \mathrm{C}$ with a Labconco Lyph-lock 1L (model 77400; Labconco Corporation). Protein samples were digested in a $10 \mu \mathrm{g} / \mathrm{ml}$ sequencing grade-modified trypsin (Promega Corporation) in $30 \%$ acetonitrile (ACN), $50 \mathrm{mM}$ ammonium bicarbonate, and $5 \mathrm{mM}$ DTT overnight at $30^{\circ} \mathrm{C}$. Digested proteins were lyophilized overnight at $4^{\circ} \mathrm{C}$ and dissolved in $0.1 \%$ formic acid. MS was performed as previously described (17).

An Agilent 1100 LC/MSD Trap XCT (Agilent Technologies, Inc.) was used for high performance liquid chromatography and MS/MS. Each sample (25 $\mu \mathrm{l})$ was used and separated in columns (Zorbax 300SB-C18; $75 \mu \mathrm{m} ; 150 \mathrm{~mm}$; Agilent Technologies, Inc.). The Agilent 1100 capillary pump (Agilent Technologies, Inc.) was operated under the following conditions: Solvent A, $0.1 \%$ formic acid; solvent $\mathrm{B}, \mathrm{ACN}$ in $0.1 \%$ formic acid; column flow, $0.3 \mu \mathrm{l} / \mathrm{min}$; primary flow, $300 \mu \mathrm{l} / \mathrm{min}$; gradient, $0-5 \mathrm{~min}$; $2 \%$ solvent B, 60 min $60 \%$ solvent B; stop time, 60 min. Protein identification was performed using the Agilent Spectrum MILL MS proteomics workbench (Rev BI.07.00.208; Agilent Technologies, Inc.) and the Swiss-Prot protein sequence database search engine (http://kr.expasy.org/sprot/) and the MASCOT MS/MS Ions search engine (http://www. matrixscience.com/search_form_select.html). The positive identification criteria of proteins were set as follows: Protein score, $>10.0$ and peak intensity score, $>8 \%$ or peptide score.

Online analysis of candidate genes. Candidate genes were analyzed using the GEPIA database and the default settings (18). The Cancer Genome Atlas (TCGA) database (https://portal.gdc.cancer.gov/) and The Broad Institute Cancer Cell Line Encyclopedia (CCLE) database (https://portals. broadinstitute.org/ccle) were screened for the potential genes with default settings; the datasets were downloaded, and the histograms were drawn using GraphPad Prism 5.0 (GraphPad Software, Inc.).

Statistical analysis. Statistical analyses were performed using GraphPad Prism 5.0. For the comparison of protein levels from MS data, all datapoints were presented, and two-tailed Student's t-test was performed to analyze the differences. For survival analysis, log-rank test, also termed the Mantel-Cox test, was performed on GEPIA. $\mathrm{P}<0.05$ was considered to indicate a statistically significant difference.

\section{Results}

Upregulated membrane proteins in pancreatic adenocarcinoma. To identify novel pancreatic cancer specific membrane proteins, total protein from seven paired PAAD and adjacent normal tissues were subjected to mass spectrum analysis, and membrane proteins were selected for further analysis. The results demonstrated that the protein expression levels of CD2, CD55, CD109, CD276, transmembrane and tetratricopeptide repeat containing 3 (TMTC3), transmembrane protein (TMEM) 70, TMEM165, TMEM179B, TMEM231 and calcium/calmodulin-dependent serine protein kinase (CASK) were the top 10 increased membrane proteins in pancreatic cancer tissues compared with that in adjacent normal tissues (Fig. 1). For further evaluation on the potential of these 10 membrane proteins, the GEPIA online database was used, so that the mRNA expression level of these membrane proteins in pancreatic cancer samples could be investigated. Notably, the mRNA expression levels of all ten membrane proteins were upregulated in PAAD samples compared with those in the adjacent normal tissues, as illustrated in Fig. 2A, indicating the validity of the mass spectrum data. Furthermore, overall survival time of patients with PAAD, included in the GEPIA database was analyzed and it was found that high protein expression levels of CASK protein in patients with PAAD was significantly associated with poor overall survival time (Fig. 2B) suggesting poor outcome of patients with PAAD and high expression levels of CASK.

Next, the mRNA expression level of CASK was analyzed in from the CCLE database and found that CASK was 
Table I. Information of the patients with pancreatic adenocarcinoma.

\begin{tabular}{llcl}
\hline Sample no. & Sex & Age, years & Pathological type \\
\hline P1 & Male & 73 & Moderately differentiated \\
P14 & Male & 45 & Moderately-poorly differentiated \\
P22 & Male & 53 & Moderately-poorly differentiated \\
P32 & Male & 75 & Moderately differentiated \\
P36 & Male & 50 & Moderately-poorly differentiated \\
P40 & Male & 50 & Poorly differentiated \\
P45 & Female & 57 & Moderately differentiated \\
\hline
\end{tabular}
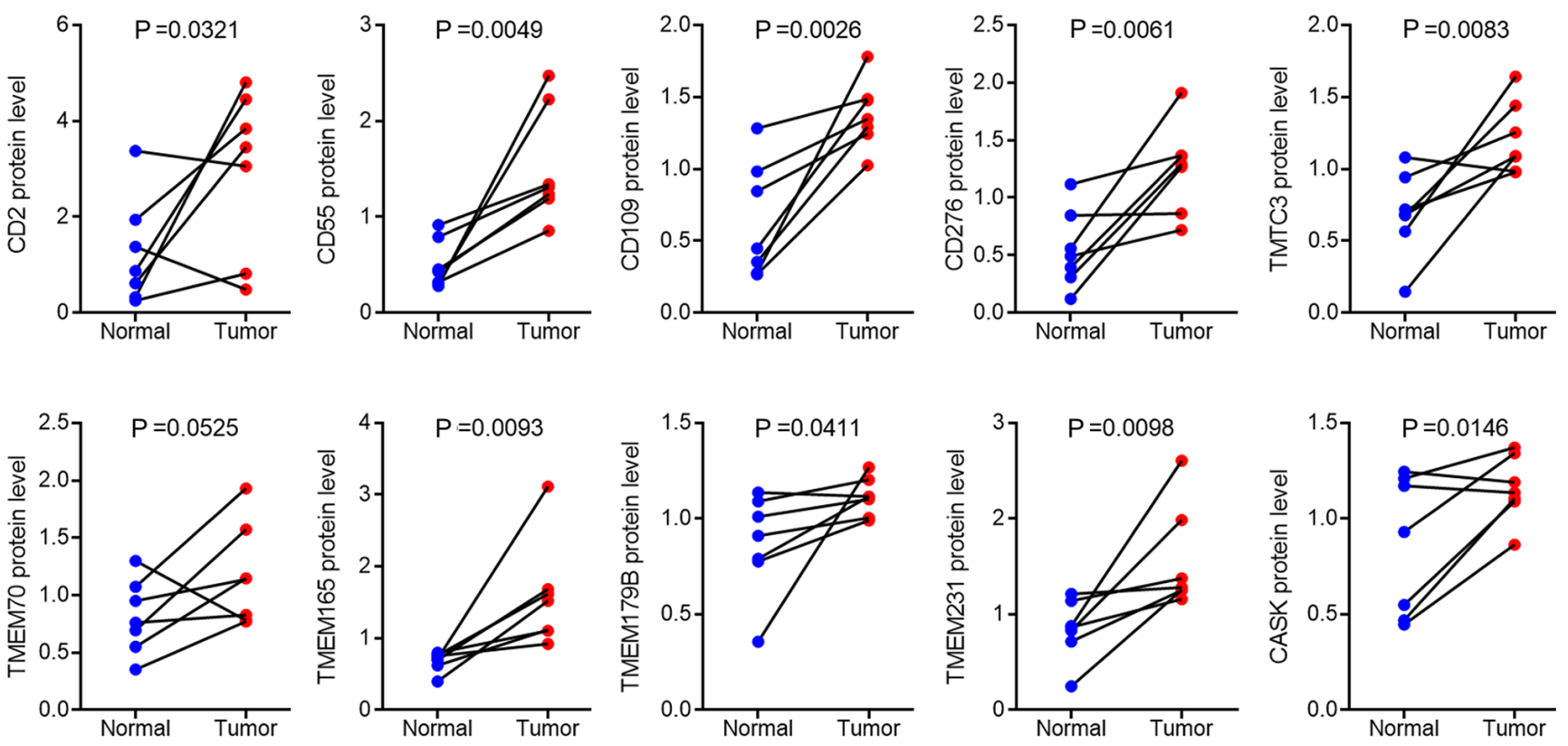

Figure 1. Upregulated membrane proteins in PAAD. The protein expression level of 10 upregulated membrane proteins revealed using mass spectrum in PAAD. PAAD, pancreatic adenocarcinoma.

upregulated in PAAD cell lines (ranking 10th) compared with that in other types of cancer cells (Fig. 3A) and the relative mRNA expression level of CASK was upregulated in most PAAD cell lines (Fig. 3B).

Taken together, the data showed that CASK was increased in PAAD samples and cell lines and was associated with of poor outcome in patients with PAAD.

Downregulated membrane proteins in pancreatic adenocarcinoma. Subsequently, the downregulated membrane proteins were analyzed in the mass spectrum data and eight proteins were significantly decreased, CD36, TMEM41B, TMEM205, TMEM214, erythrocyte membrane protein band 4.2 (EPB42), family with sequence similarity 174 member B (FAM174B), transmembrane and ubiquitin-like domain-containing 1 (TMUB1), and vesicle-associated membrane protein 5 (VAMP5) in pancreatic cancer tissues compared with that in adjacent normal tissues, as illustrated in Fig. 4. Following validation of the mRNA expression level of these genes using the GEPIA database, it was found that the mRNA expression levels of these genes were not significantly downregulated in PAAD samples compared with normal tissues (Fig. 5A); however, it was observed the mRNA levels of TMEM205 and VAMP5 were upregulated in PAAD, which suggested a complicated posttranscriptional regulation of these potential membrane markers in PAAD. Notably, low expression level of CD36 or EPB42 was associated with poor overall survival of patients with PAAD (Fig. 5B) suggesting that the reduction of CD36 or EPB42 was associated with poor outcome in patients in PAAD.

Furthermore, the mRNA expression level of CD36 and EPB42 in the CCLE database was investigated and it was found that both CD36 or EPB42 were decreased in PAAD cell lines compared with that in other types of cancer cell lines (Figs. 6A and 7A, respectively). Furthermore, the mRNA expression levels of CD36 and EPB42 were decreased in most PAAD cell lines (Figs. 6B and 7B, respectively). Therefore, the results in the present study suggested that the downregulation of CD36 and EPB42 in PAAD samples predicted poorer survival outcomes in patients with PAAD, and upregulation of CASK predicted poorer survival outcomes in patients with PAAD. 
A
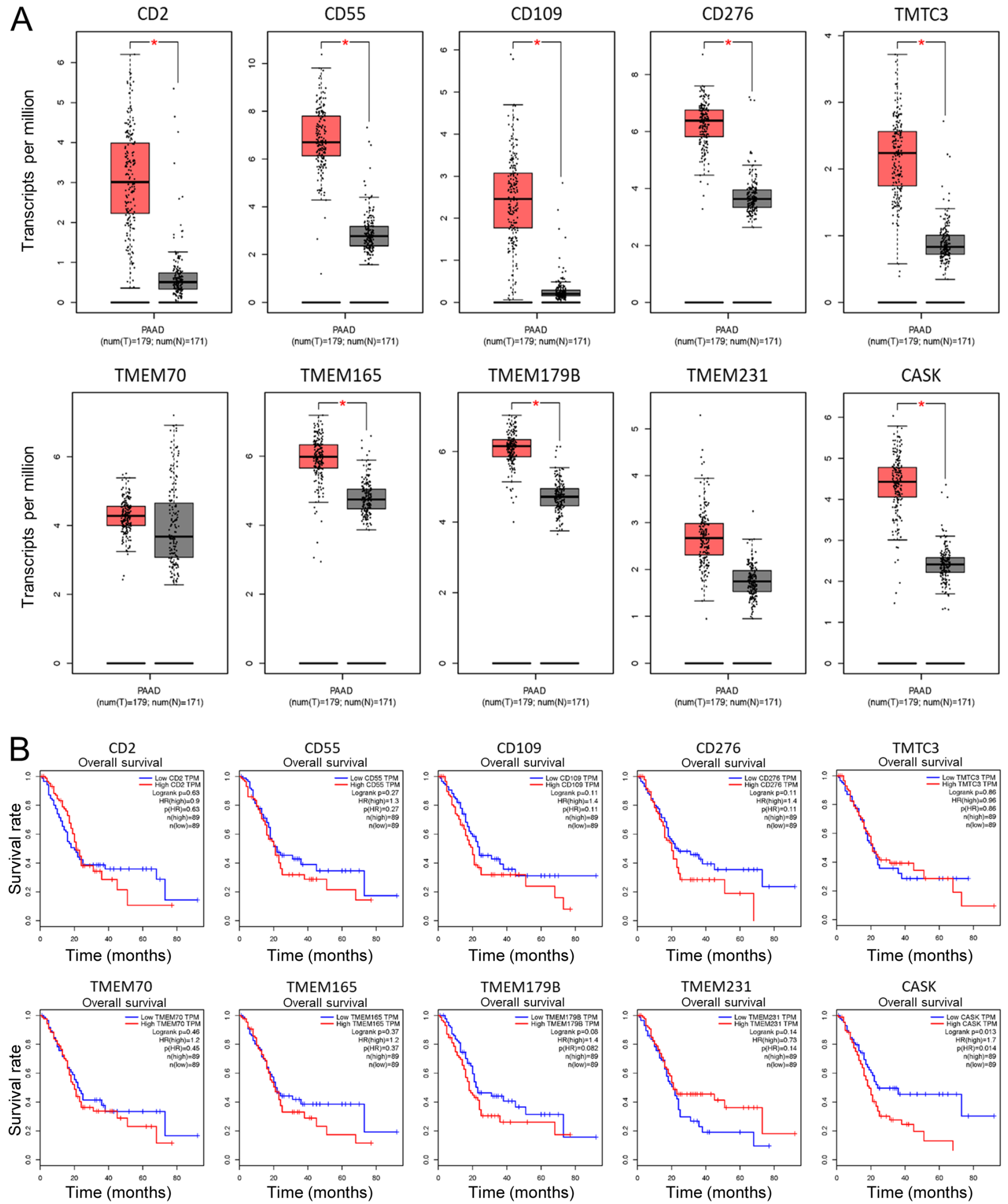

Figure 2. mRNA expression analysis of the upregulated membrane proteins in PAAD. (A) mRNA expression levels and (B) association between patient overall survival time and the mRNA expression level of the 10 upregulated membrane proteins from the GEPIA database. *P<0.05. Red, tumor tissue; grey, normal tissue. PAAD, pancreatic adenocarcinoma; T, tumor; N, normal.

\section{Discussion}

Pancreatic cancer exhibits a highly fatal malignancy, which obstructs current immunotherapy (10). In the present study,
18 aberrantly expressed cell surface proteins were identified in seven paired PAAD and adjacent normal tissues. Further analysis using an online database validated the mass spectrum results, even without performing large-scale benchwork 
$\mathrm{A}_{6}$
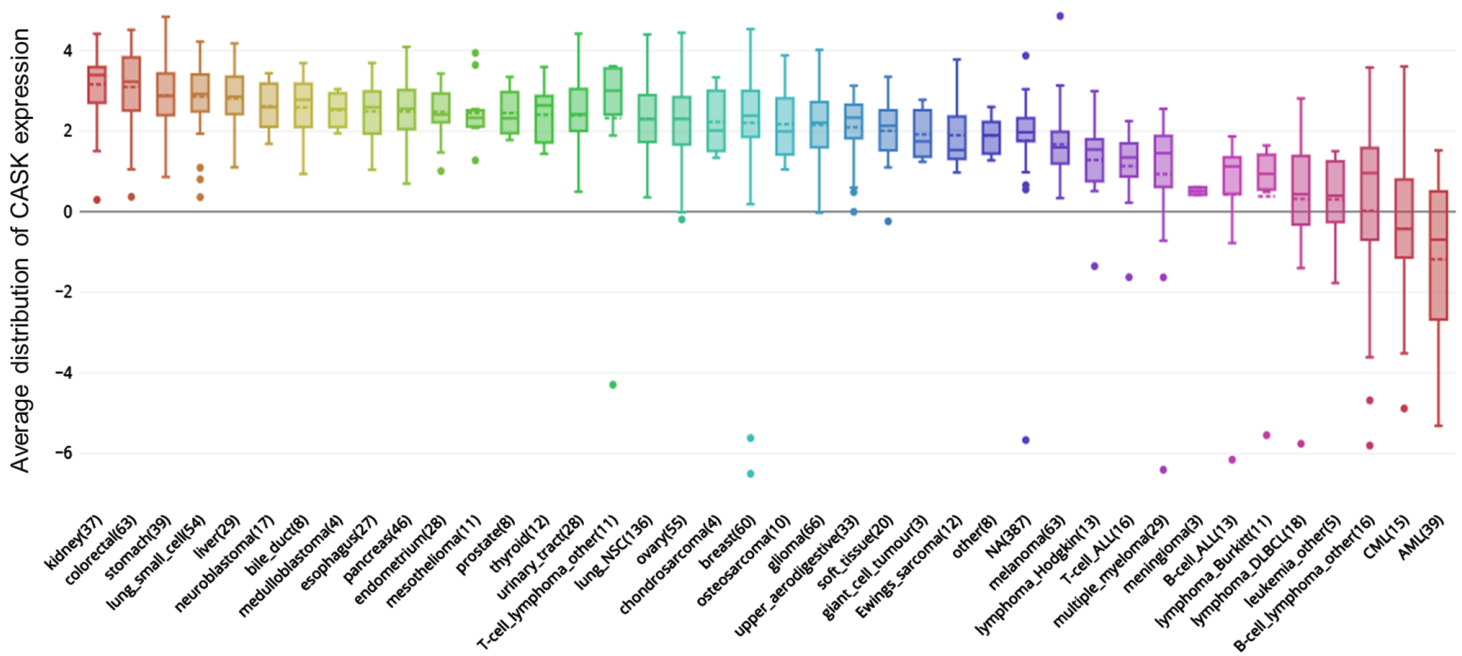

B
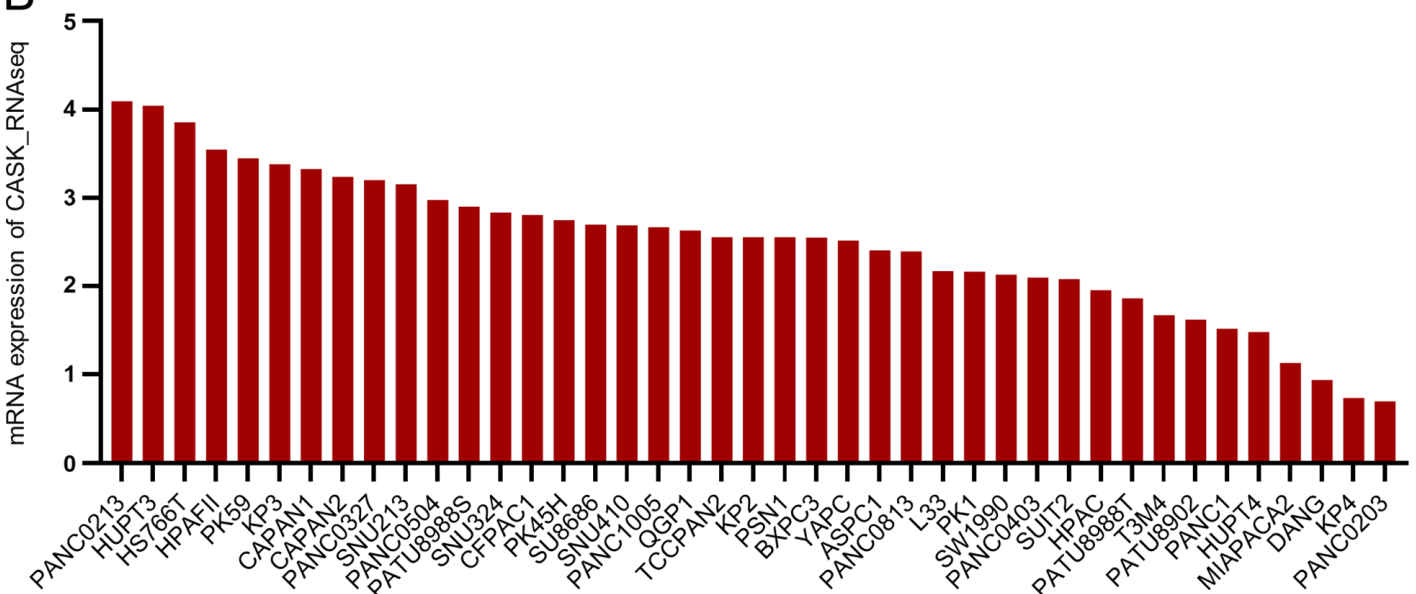

Figure 3. mRNA expression level of CASK in various types of cancer and pancreatic cancer cell lines. mRNA expression level of CASK in (A) various types of cancer and (B) various types of pancreatic cancer cell lines revealed using the Broad Institute Cancer Cell Line Encyclopedia database. Seq, sequencing.
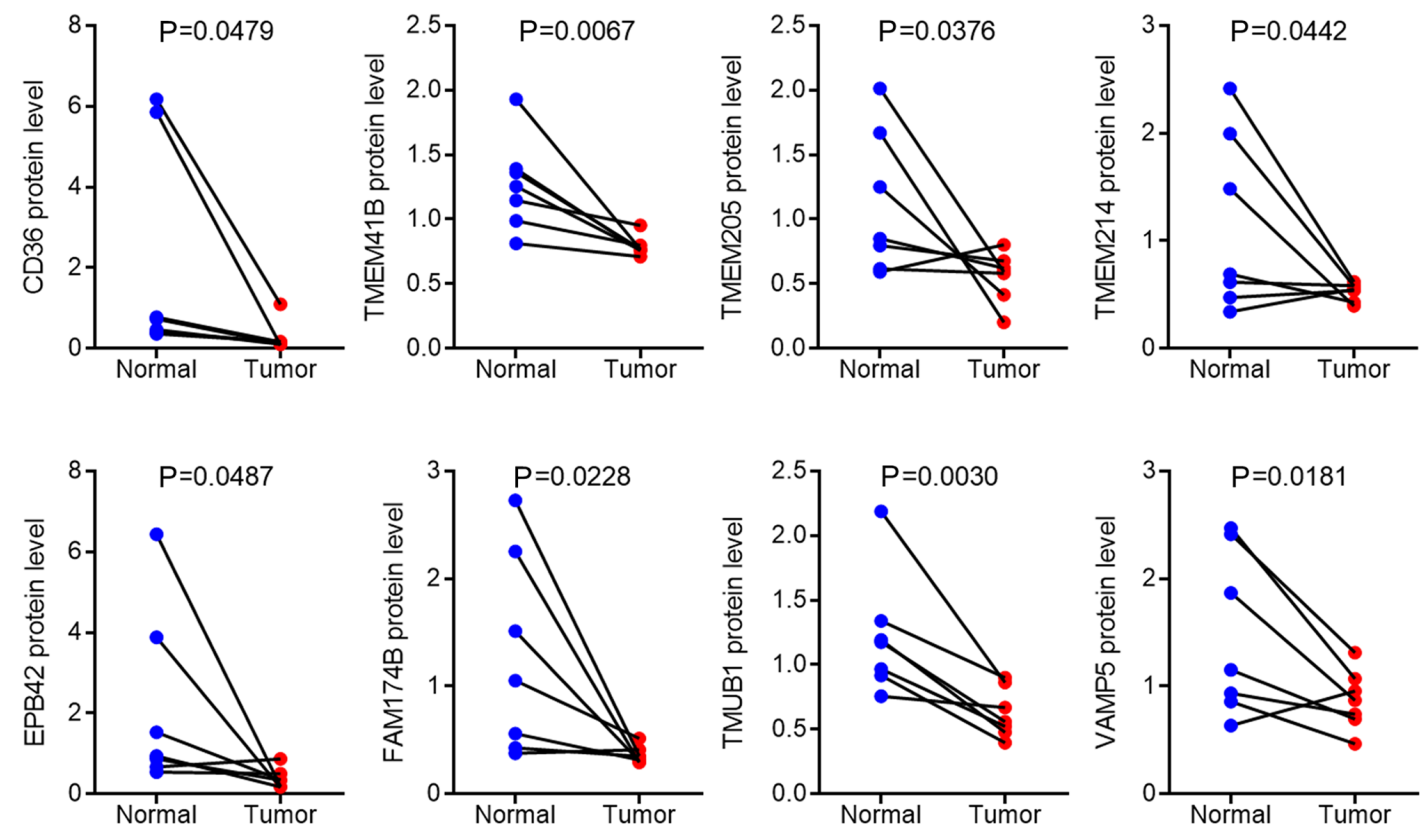

Figure 4. Downregulated membrane proteins in PAAD. The protein expression level of 8 downregulated membrane proteins revealed using mass spectrum in PAAD. PAAD, pancreatic adenocarcinoma. 

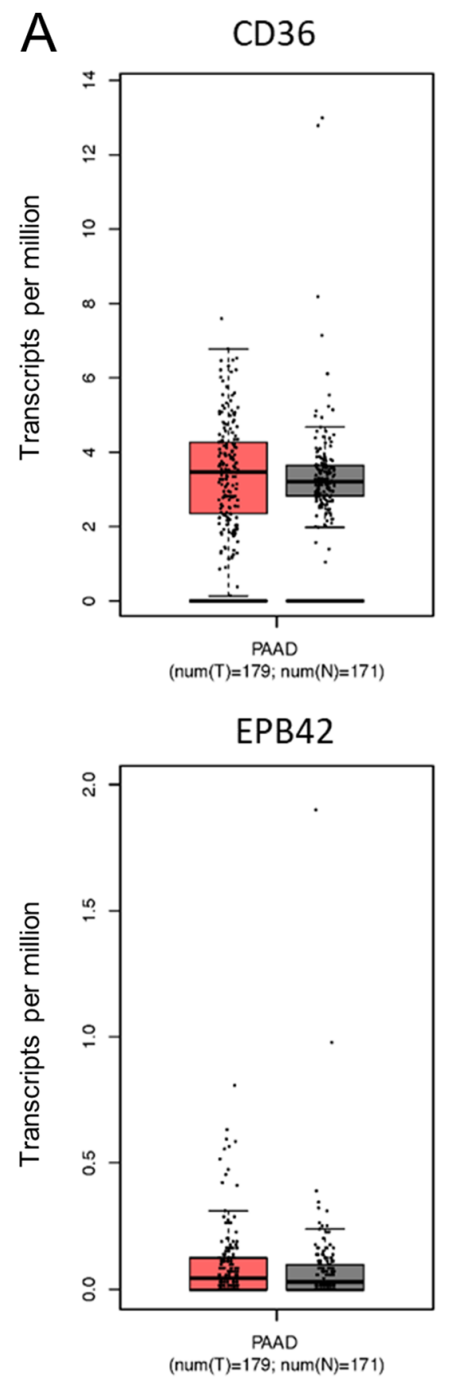

TMEM41B

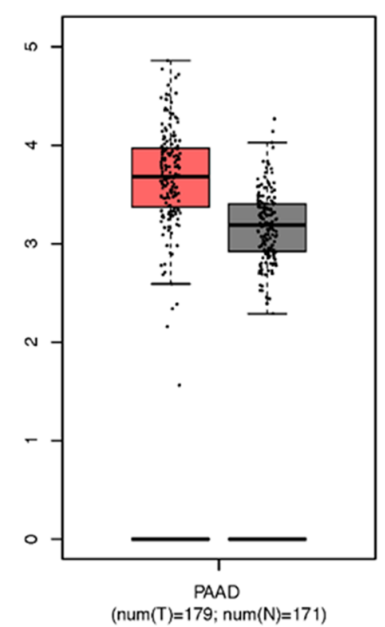

FAM174B

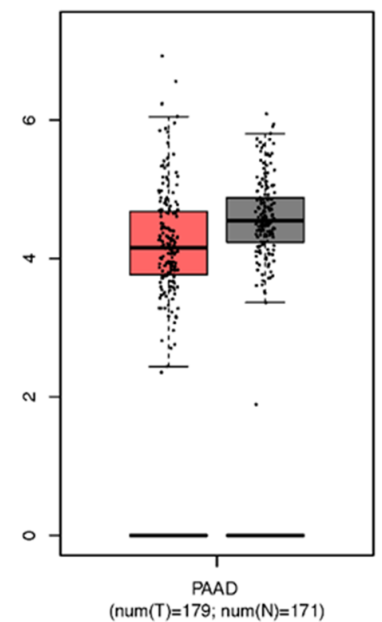

TMEM205

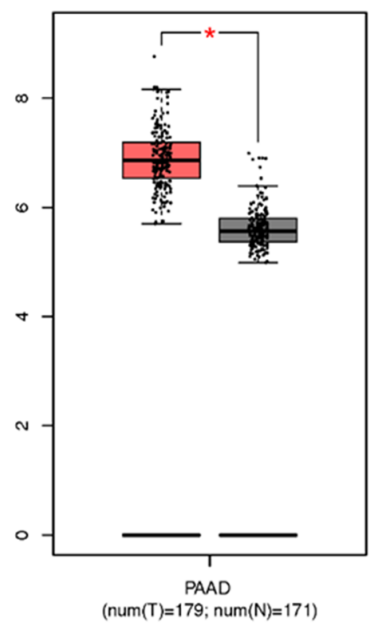

TMUB1

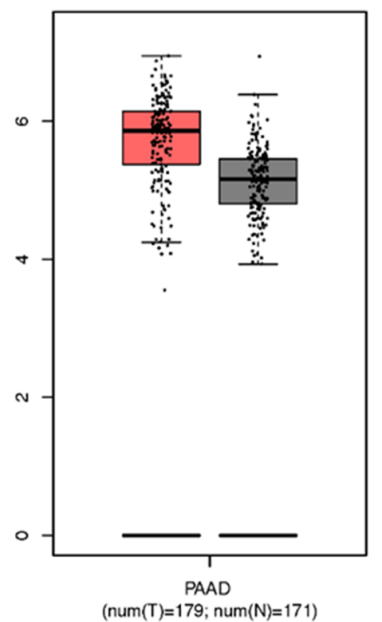

TMEM214

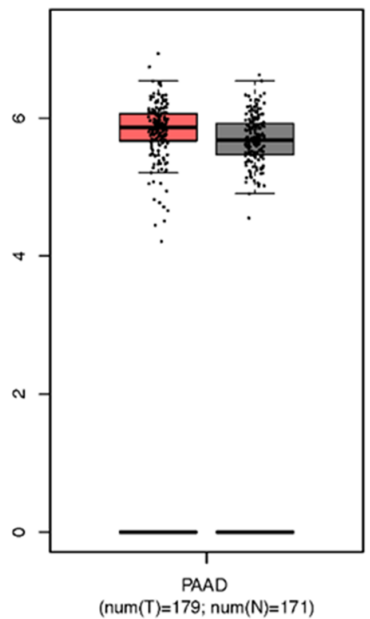

VAMP5

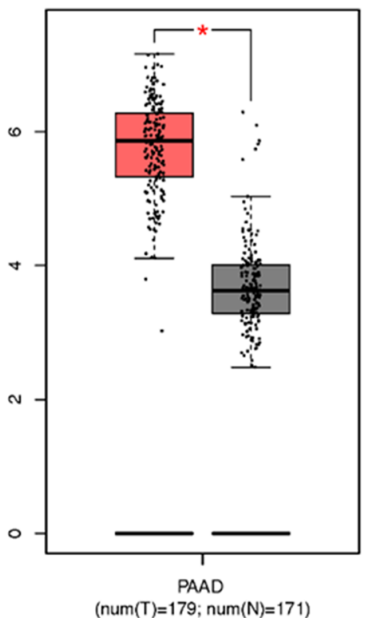

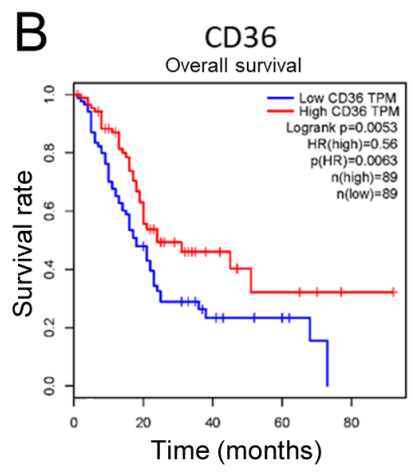
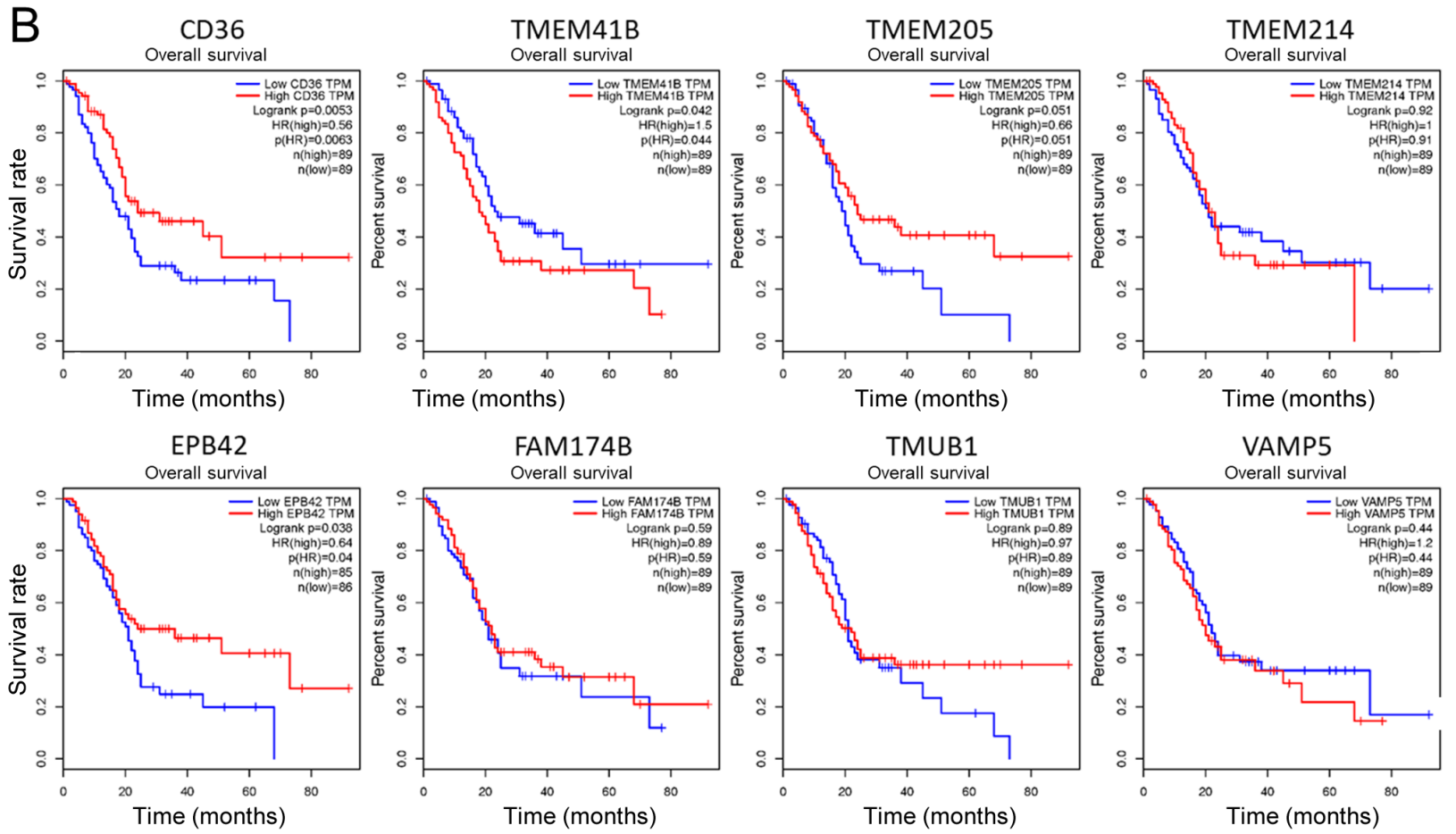

Figure 5. mRNA expression analysis of the downregulated membrane proteins in PAAD. (A) mRNA expression levels and (B) association between patient overall survival time and mRNA expression levels of the eight downregulated membrane proteins from the GEPIA database. "P<0.05. Red, tumor tissue; grey, normal tissue. PAAD, pancreatic adenocarcinoma; $\mathrm{T}$, tumor; $\mathrm{N}$, normal. 

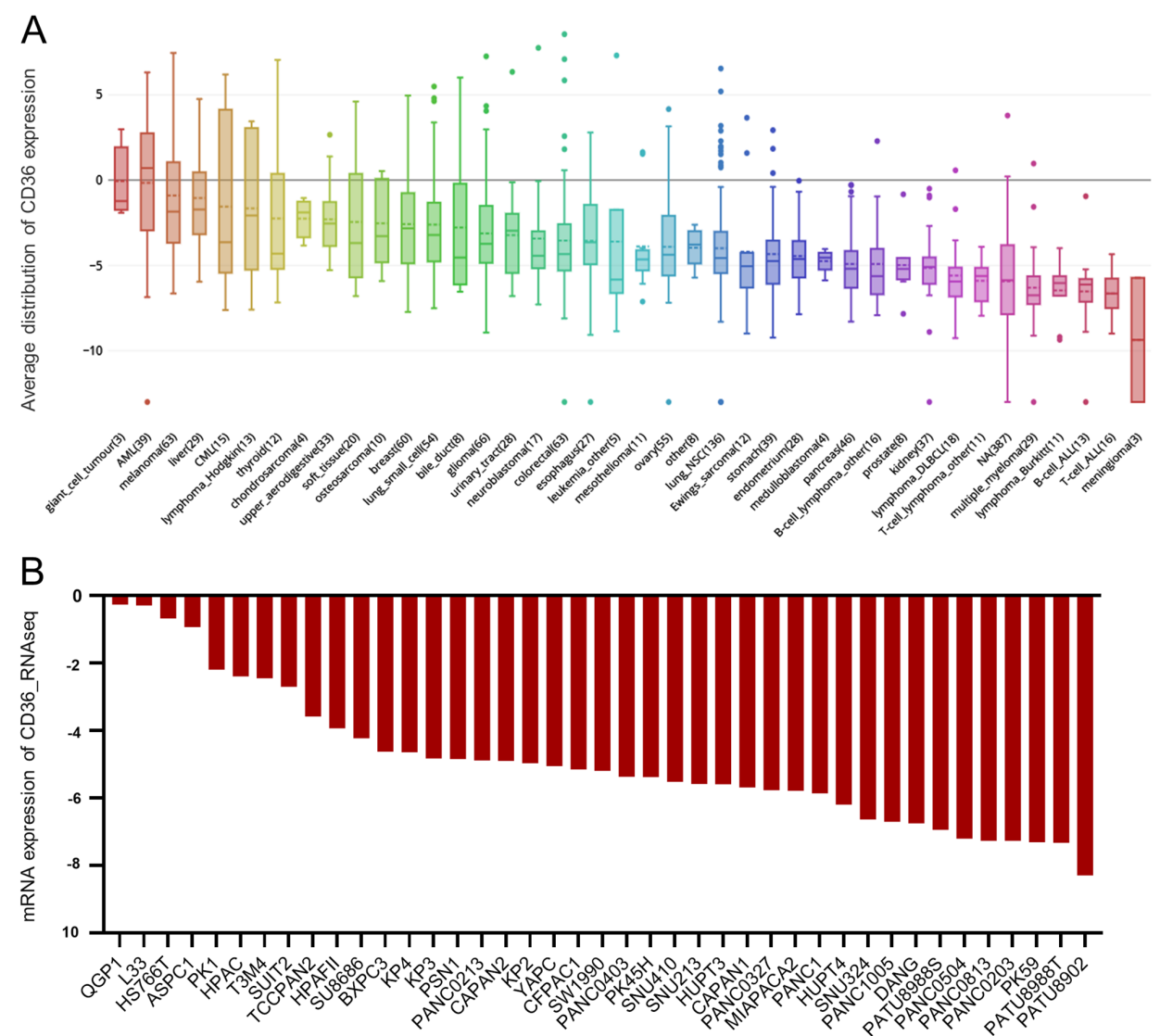

Figure 6. mRNA expression level of CD36 in various types of cancer and pancreatic cancer cell lines. mRNA expression level of CD36 in (A) various types of cancer and (B) various types of pancreatic cancer cell lines revealed using the Broad Institute Cancer Cell Line Encyclopedia database. Seq, sequencing.

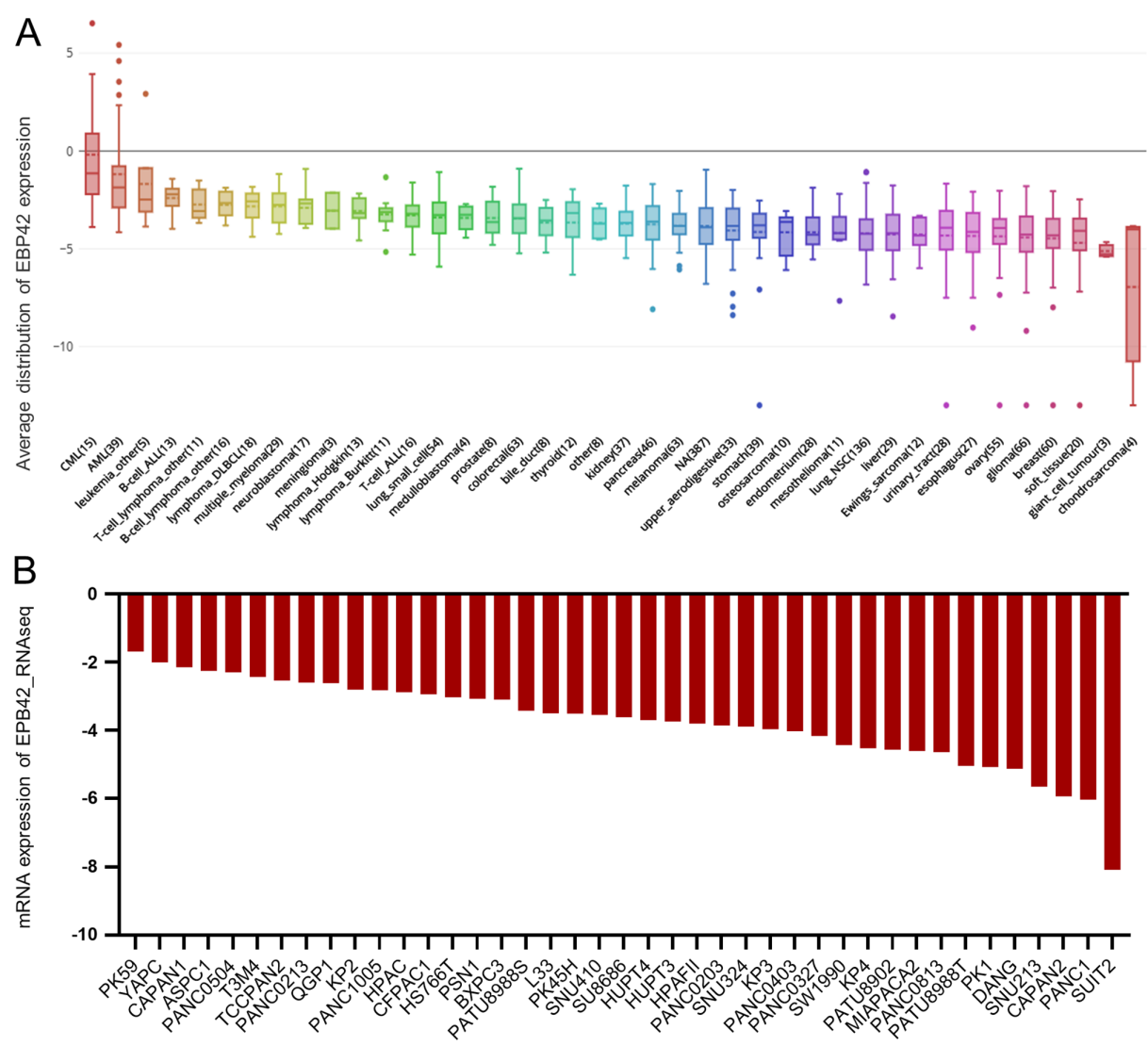

Figure 7. mRNA expression level of EBP42 in various types of cancer and pancreatic cancer cell lines. mRNA expression level of EBP42 in (A) various types of cancer and (B) various types of pancreatic cancer cell lines revealed using the Broad Institute Cancer Cell Line Encyclopedia database. Seq, sequencing. 
validations. Among the upregulated membrane proteins, there was a significant increase in CASK in patients with PAAD, revealed by the online database. CASK was initially identified as an interactor of neurexins in neuronal cells (19). It has been established that CASK is a membrane-associated guanylate kinase, which can enter the nucleus and acts as a coactivator to induce transcription of cerebrocortical development-related genes, and whose mutation resulted in microcephaly and hypoplasia of the brainstem and cerebellum $(20,21)$. Notably, a previous study showed that CASK and its target gene, reelin, were co-upregulated in human esophageal carcinoma (22). Furthermore, it has been proven that CASK was increased in gastric cancer cells and promoted their proliferation and invasion (23). Therefore, it is important to further investigate the role of CASK in pancreatic cancer carcinogenesis.

The results in the present study also demonstrated the increase of CD2 in PAAD samples, while a recent report investigated the dose escalation of the anti-CD2 monoclonal antibody in aggressive peripheral T-cell lymphomas (24). Although in the present study the upregulation of CD2 was not associated with survival, testing this antibody may be significant for the treatment of pancreatic cancer since it provides novel insights into immunotherapy. Furthermore, it was found that CD55 was increased in patients with pancreatic cancer recruited into the study and from the online database. It has been recently proposed that CD55 regulated anticancer drug resistance in endometrioid tumors (25) and cervical cancer (26). All these data suggested an oncogenic role of the membrane protein CD55. Notably, CD276 (B7-H3), which is an important immune checkpoint member of the B7 and CD28 families (26), was also increased in patients with pancreatic cancer. It has been shown that CD276 protein expression was increased in pancreatic cancer tissues and CD276 could block CD8 ${ }^{+}$T-cell infiltration to induce an antitumor effect (27). The results from the present study provided independent evidence that CD276 was upregulated in PAAD and further strengthened the possibility to develop immunotherapy and chemotherapy by targeting CD276. Furthermore, CD109, TMTC3, TMEM165, and TMEM179B were also increased in PAAD despite their roles in cancer being elusive. Therefore, these targets may serve as novel diagnostic markers and therapeutic targets when appropriate validations are provided.

In addition to the upregulated membrane proteins, eight downregulated membrane proteins were identified in patients with PAAD. TMEM205 was significantly downregulated in PAAD, and it has been reported that TMEM205 was associated with cisplatin resistance in PAAD $(28,29)$. However, the genuine function of TMEM205 requires further investigation. The mass spectrum data revealed that VAMP5, the function of which in cancer is still largely unknown, was downregulated in PAAD samples. It is important to further dissect the biological function of TMEM205 in PAAD cell lines. Notably, the results in the present study revealed the decrease of CD36 and EPB42 in the seven patients with PAAD, while low expression level of CD36 and EPB42 was associated with poor outcome in patients with PAAD. CD36 is a critical fatty acid receptor, which initiates the metastasis of human oral carcinomas (30). Importantly, silencing of CD36 also impaired metastasis in human melanoma- and breast cancer-derived tumors (30). With respect to the mRNA and protein expression levels of CD36 in PAAD, revealing the role of $\mathrm{CD} 36$ in PAAD metastasis may provide a novel strategy for PAAD treatment. The inconsistent expression pattern of CD36 may result from the variety of patient samples. Further in-depth analysis is required to characterize the role of CD36. On the other hand, the role of EPB42, a crucial protein in hereditary spherocytosis (31), in cancer is less well-known. The mRNA expression levels of CD36 and EPB42 were not statistically significantly decreased from the GEPIA database; however, this could be due to post-transcriptional regulation. In addition, a larger cohort of samples from different databases may also be required to validate the results.

In summary, the results of the present study identified multiple aberrantly expressed membrane proteins in patients with PAAD, which may provide novel diagnostic markers and drug targets for the immunotherapy of pancreatic cancer.

\section{Acknowledgements}

Not applicable.

\section{Funding}

This study was supported by the National Natural Science Foundation of China (grant no. 81772798).

\section{Availability of data and materials}

The datasets used and/or analyzed during the present study are available from the corresponding author on reasonable request.

\section{Authors' contributions}

MM, CW, XG, SL, XX and ELH performed the literature research. $\mathrm{MM}, \mathrm{XG}, \mathrm{XX}$ and $\mathrm{ELH}$ performed the clinical studies. MM, CW, SL and XX performed the experiments. MM, CW, SL and ELH performed statistical analysis. MM, CW, XX and ELH edited the manuscript. SL and ELH were guarantors of the integrity of the study. All authors read and approved the final manuscript.

\section{Ethics approval and consent to participate}

All patients involved in the study provided written informed consent, and all procedures were approved by the Ethics Committee of Chinese PLA General Hospital in accordance with the Declaration of Helsinki.

\section{Patient consent for publication}

Not applicable.

\section{Competing interests}

The authors declare that they have no competing interests.

\section{References}

1. Barkal AA, Brewer RE, Markovic M, Kowarsky M, Barkal SA Zaro BW, Krishnan V, Hatakeyama J, Dorigo O, Barkal LJ and Weissman IL: CD24 signalling through macrophage Siglec-10 is a target for cancer immunotherapy. Nature 572: 392-396, 2019. 
2. Gao A, Hu XL, Saeed M, Chen BF, Li YP and Yu HJ: Overview of recent advances in liposomal nanoparticle-based cancer immunotherapy. Acta Pharmacol Sin 40: 1129-1137, 2019.

3. Eroglu Z, Zaretsky JM, Hu-Lieskovan S, Kim DW, Algazi A, Johnson DB, Liniker E, Kong B, Munhoz R, Rapisuwon S, et al: High response rate to PD-1 blockade in desmoplastic melanomas. Nature 553: 347-350, 2018.

4. Jiang XM, Xu YL, Huang MY, Zhang LL, Su MX, Chen X and Lu JJ: Osimertinib (AZD9291) decreases programmed death ligand-1 in EGFR-mutated non-small cell lung cancer cells. Acta Pharmacol Sin 38: 1512-1520, 2017.

5. Rizvi NA, Hellmann MD, Snyder A, Kvistborg P, Makarov V, Havel JJ, Lee W, Yuan J, Wong P, Ho TS, et al: Cancer immunology. Mutational landscape determines sensitivity to PD-1 blockade in non-small cell lung cancer. Science 348: 124-128, 2015.

6. Royal RE, Levy C, Turner K, Mathur A, Hughes M, Kammula US, Sherry RM, Topalian SL, Yang JC, Lowy I and Rosenberg SA: Phase 2 trial of single agent Ipilimumab (anti-CTLA-4) for locally advanced or metastatic pancreatic adenocarcinoma. J Immunother 33: 828-833, 2010.

7. Thind K, Padrnos LJ, Ramanathan RK and Borad MJ: Immunotherapy in pancreatic cancer treatment: A new frontier. Therap Adv Gastroenterol 10: 168-194, 2017.

8. Bayne LJ, Beatty GL, Jhala N, Clark CE, Rhim AD, Stanger BZ and Vonderheide RH: Tumor-derived granulocyte-macrophage colony-stimulating factor regulates myeloid inflammation and $\mathrm{T}$ cell immunity in pancreatic cancer. Cancer Cell 21: 822-835, 2012.

9. Özdemir BC, Pentcheva-Hoang T, Carstens JL, Zheng X, Wu CC, Simpson TR, Laklai H, Sugimoto H, Kahlert C, Novitskiy SV, et al: Depletion of carcinoma-associated fibroblasts and fibrosis induces immunosuppression and accelerates pancreas cancer with reduced survival. Cancer Cell 25: 719-734, 2014.

10. Panni RZ, Herndon JM,Zuo C, Hegde S, Hogg GD, Knolhoff BL, Breden MA, Li X, Krisnawan VE, Khan SQ, et al: Agonism of CD11b reprograms innate immunity to sensitize pancreatic cancer to immunotherapies. Sci Transl Med 11: eaau9240, 2019.

11. Jiang H, Hegde S, Knolhoff BL, Zhu Y, Herndon JM, Meyer MA, Nywening TM, Hawkins WG, Shapiro IM, Weaver DT, et al: Targeting focal adhesion kinase renders pancreatic cancers responsive to checkpoint immunotherapy. Nat Med 22: 851-860, 2016.

12. Mace TA, Shakya R, Pitarresi JR, Swanson B, McQuinn CW, Loftus S, Nordquist E, Cruz-Monserrate Z, Yu L, Young G, et al: IL-6 and PD-L1 antibody blockade combination therapy reduces tumour progression in murine models of pancreatic cancer Gut 67: 320-332, 2018.

13. Zhou L, Husted H, Moore T, Lu M, Deng D, Liu Y, Ramachandran V, Arumugam T, Niehrs C, Wang $\mathrm{H}$, et al: Suppression of stromal-derived Dickkopf-3 (DKK3) inhibits tumor progression and prolongs survival in pancreatic ductal adenocarcinoma. Sci Transl Med 10: eaat3487, 2018.

14. Melo SA, Luecke LB, Kahlert C, Fernandez AF, Gammon ST, Kaye J, LeBleu VS, Mittendorf EA, Weitz J, Rahbari N, et al: Glypican-1 identifies cancer exosomes and detects early pancreatic cancer. Nature 523: 177-182, 2015.

15. Bydoun M, Sterea A, Liptay H, Uzans A, Huang WY Rodrigues GJ, Weaver ICG, Gu H and Waisman DM: S100A10, a novel biomarker in pancreatic ductal adenocarcinoma. Mol Oncol 12: 1895-1916, 2018.

16. Tempero MA, Malafa MP, Chiorean EG, Czito B, Scaife C, Narang AK, Fountzilas C, Wolpin BM, Al-Hawary M, Asbun H, et al: Pancreatic adenocarcinoma, version 1.2019. J Natl Compr Canc Netw 17: 202-210, 2019.
17. Kuramitsu Y, Taba K, Ryozawa S, Yoshida K, Zhang X, Tanaka T, Maehara SI, Maehara Y, Sakaida I and Nakamura K: Identification of up- and down-regulated proteins in gemcitabine-resistant pancreatic cancer cells using two-dimensional gel electrophoresis and mass spectrometry. Anticancer Res 30: 3367-3372, 2010.

18. Tang Z, Li C, Kang B, Gao G, Li C and Zhang Z: GEPIA: A web server for cancer and normal gene expression profiling and interactive analyses. Nucleic Acids Res 45W: W98-W102, 2017.

19. Hata Y, Butz S and Südhof TC. Sudhof, CASK: A novel dlg/PSD95 homolog with an N-terminal calmodulin-dependent protein kinase domain identified by interaction with neurexins. J Neurosci 16: 2488-2494, 1996.

20. Hsueh YP, Wang TF, Yang FC and Sheng M: Nuclear translocation and transcription regulation by the membrane-associated guanylate kinase CASK/LIN-2. Nature 404: 298-302, 2000.

21. Najm J, Horn D, Wimplinger I, Golden JA, Chizhikov VV, Sudi J, Christian SL, Ullmann R, Kuechler A, Haas CA, et al: Mutations of CASK cause an X-linked brain malformation phenotype with microcephaly and hypoplasia of the brainstem and cerebellum. Nat Genet 40: 1065-1067, 2008.

22. Wang Q, Lu J, Yang C, Wang X, Cheng L, Hu G, Sun Y, Zhang X, Wu M and Liu Z: CASK and its target gene Reelin were co-upregulated in human esophageal carcinoma. Cancer Lett 179: 71-77, 2002.

23. Zhou X, Xu G, Yin C, Jin W and Zhang G: Down-regulation of miR-203 induced by Helicobacter pylori infection promotes the proliferation and invasion of gastric cancer by targeting CASK. Oncotarget 5: 11631-11640, 2014.

24. Roswarski J, Roschewski M, Lucas A, Melani C, Pittaluga S, Jaffe ES, Steinberg SM, Waldmann TA and Wilson WH: Phase I dose escalation study of the anti-CD2 monoclonal antibody, siplizumab, with DA-EPOCH-R in aggressive peripheral T-cell lymphomas. Leuk Lymphoma 59: 1466-1469, 2018.

25. Saygin C, Wiechert A, Rao VS, Alluri R, Connor E, Thiagarajan PS, Hale JS, Li Y, Chumakova A, Jarrar A, et al: CD55 regulates self-renewal and cisplatin resistance in endometrioid tumors. J Exp Med 214: 2715-2732, 2017.

26. Leung TH, Tang HW, Siu MK, Chan DW, Chan KK, Cheung AN and Ngan HY: Human papillomavirus E6 protein enriches the CD55(+) population in cervical cancer cells, promoting radioresistance and cancer aggressiveness. J Pathol 244: 151-163, 2018.

27. Yamato I, Sho M, Nomi T, Akahori T, Shimada K, Hotta K, Kanehiro H, Konishi N, Yagita H and Nakajima Y: Clinical importance of B7-H3 expression in human pancreatic cancer. $\mathrm{Br}$ J Cancer 101: 1709-1716, 2009.

28. Shen DW and Gottesman MM: RAB8 enhances TMEM205mediated cisplatin resistance. Pharm Res 29: 643-650, 2012.

29. Shen DW, Ma J, Okabe M, Zhang G, Xia D and Gottesman MM: Elevated expression of TMEM205, a hypothetical membrane protein, is associated with cisplatin resistance. J Cell Physiol 225: 822-828, 2010.

30. Pascual G, Avgustinova A, Mejetta S, Martín M, Castellanos A, Attolini CS, Berenguer A, Prats N, Toll A, Hueto JA, et al: Targeting metastasis-initiating cells through the fatty acid receptor CD36. Nature 541: 41-45, 2017.

31. Kanzaki A, Hayette S, Morlé L, Inoue F, Matsuyama R, Inoue T, Yawata A, Wada H, Vallier A, Alloisio N, et al: Total absence of protein 4.2 and partial deficiency of band 3 in hereditary spherocytosis. Br J Haematol 99: 522-530, 1997.

This work is licensed under a Creative Commons Attribution-NonCommercial-NoDerivatives 4.0 International (CC BY-NC-ND 4.0) License. 